\title{
UNCERTAINTY ESTIMATION OF EXTERNAL BLAST EFFECTS USING THE MONTE-CARLO METHOD
}

\author{
GABRIEL SEISSON, THIERRY LACAZE \& ALAIN ROUQUAND \\ CEA/Gramat, France
}

\begin{abstract}
Protection against terrorist assaults is a major concern for many countries all over the world. It entails developing counter-terrorism intelligence and intervention means, allowing the avoidance of such events but also to be able to rapidly (but efficiently) predict the structural effects of attacks on buildings. In previous works carried out at CEA/Gramat, an analytical approach was developed to promptly obtain the response of a rectangular masonry panel submitted to the blast effects of an aerial explosion. In this approach, the panel was simulated as a single-degree-of-freedom system with an elastic and perfectly plastic behaviour and the blast loading was reduced to a triangular pressure profile. The purpose was to predict either the failure of the panel or its survival. Besides, free-field experiments and corresponding FEM calculations led one to identify sets of parameters for various structural materials such as concrete, cinderblocks and bricks. The present study aims to enhance the binary result of the model towards an interval of failure/survival probability with a confidence level. Through the example of a unique generic wall, we present the modelling of the uncertainties in both geometric and material parameters, the use of the Monte-Carlo method, and the interpretation of the obtained probability density functions. A sensitivity analysis emphasizes the parameters for which a better insight is needed. Finally, a practical case of an isolated building with multiple walls is examined. Hypothesis and results are discussed and potential improvements are considered.
\end{abstract}

Keywords: blast, masonry strength, failure probability, fast running engineering model, Monte-Carlo, sensitivity analysis.

\section{INTRODUCTION}

The evaluation of aerial explosion blast effects onto buildings is a complex matter usually studied with the help of descriptive numerical finite elements simulations. This time-consuming approach requires a good knowledge of the characteristics of the structure and the explosive charge because only a few of these heavy computations will be run. In the emergency case of a malicious threat, the descriptive approach is evidently not quick enough to reliably gauge an evacuation area before the explosion.

That is why previous vulnerability studies conducted at CEA/Gramat led to the development of a fast-running engineering model (FREM) simulating a masonry panel submitted to the aerial effects of an explosion as a single-degree-of-freedom (SDOF) system loaded by a triangular pressure profile. The main eqns of the model are briefly clarified in Section 2. The identification of the parameters, based on comparisons between experimental results and finite element method (FEM) computations, is also explained.

However all the parameters of the problem are not always perfectly known. For example, if the building and its blueprints are inaccessible, the dimensions of its masonry panels will be unlikely to be precisely estimated. Even if the construction material is known, its properties can be rather different from those of the same material used for another building. A lack of knowledge may also exist about the characteristics of the explosive charge. Given all these uncertainties and others, a vulnerability study cannot reasonably lead to a clear-cut result. Thus, the Monte-Carlo method has been used to propagate uncertainties through the FREM, a work described in Section 2. 
Section 3 gives an example of our approach on a unique plain-brick panel. An effort is made on the interpretation of the results insisting on the difference between deterministic and stochastic results. Thanks to a Morris' analysis, the most sensitive parameters have been identified and the effects of the reduction of their respective uncertainties are underlined.

Finally, Section 4 presents an example of a three-floor isolated building composed of multiple walls for which the vulnerability assessment has to be inquired. The analysis of the results allowed one to determine a security perimeter for an accepted probability of damage associated with a given confidence level.

\section{ANALYTICAL MODELING}

\subsection{Masonry panel as a SDOF system}

The analytical model used in this study is inspired by the work in references [1]-[3]. It gives the Pressure Impulse diagram of an elastic and perfectly plastic SDOF system subjected to a triangular load. The analytical P-I diagram is then extended to masonry panels. The rectangular panel response is supposed to be elastic when the panel deflection is lower than the elastic deflection $x_{e}$. Between the elastic deflection $x_{e}$ and the maximum panel deflection $x_{\max }$, the panel behaviour is supposed to be perfectly plastic. The panel fails when its maximum deflection $x_{\max }$ is reached. The material ductility $\delta$ is defined by:

$$
\frac{x_{\max }}{x_{e}}=\delta
$$

The maximum deflection $x_{\max }$ is deduced on the one hand from the characteristics of the wall (mechanical behaviour, dimensions and boundary conditions) and, on the other hand from the loading level (pressure and impulse) following the expression

$$
I=\frac{\sigma_{\max } \sqrt{\frac{\rho}{E}} e}{c_{L / l}^{i} d^{i}\left(1+\frac{l^{2}}{L^{2}}\right)}\left(\frac{\left(2 x_{\max }-x_{e}\right)}{x_{e}}\right)^{1 / 2}\left[1+\frac{c^{1 / b}}{\left(\frac{c_{L / l}^{i} P l^{2}}{\sigma_{\max e^{2}\left(1-\frac{x_{e}}{2 x_{\max }}\right.}}-1\right)^{a / b}}\right],
$$

whilst the elastic deflection $x_{e}$ depends only on the panel characteristics:

$$
x_{e}=\frac{a_{L / l}^{i}}{c_{L / l}^{i}} \sigma_{\max } \frac{l^{2}}{E e} .
$$

In the above eqns, $P$ and $I$ are the pressure and impulse born by the masonry panel. Their values are calculated interpolating abacus from US Department of Defense [4] with respect to the explosive mass and to distance and angle between the panel and the explosive. $\sigma_{\max }, \rho$ and $E$ are the maximum admissible stress of the panel's material, its density and its elastic modulus, respectively. $e$ is the thickness of the masonry whereas $L$ and $l$ are its longest and shortest dimensions. $a, b$ and $c$ are coefficients depending only on the ratio $x_{\max } / x_{e}$. Boundary conditions at the four sides of the panel are taken into account by the parameters $a_{L / l}^{i}, c_{L / l}^{i}$ and $d^{i}$ where the index $L / l$ implies a dependence on this ratio.

Finally, it may be noted that, eqn (2) being irreversible according to $x_{\max }$, a zero-finder numerical algorithm such as Newton's is needed to identify the maximum deflection for a given pressure-impulse couple. 


\subsection{Identification method for the model parameters}

A specific procedure has been developed in order to identify the panel material parameters $\sigma_{\max }, E, x_{e}$ and $\delta$ that is outlined for plain bricks in the following. As a first step, experimental tests are performed on masonry walls submitted to the blast effects of an explosive charge. In these experiments the wall edges are rigidly maintained by a massive steel frame in such a way that edge rotations are impossible. Several tests are carried out and the distance between the charge and the wall is reduced in order to increase the damage level. In the last test, the wall is highly damaged and debris are thrown away from the wall.

In a second step, advanced FEM simulations are performed using ABAQUS explicit code. The blast loading is simulated using the Conwep module [5]. Here, a $\mathrm{M}_{1}-\mathrm{kg}$ spherical charge detonates at $0.6 \mathrm{~m}$ from the wall front face the dimensions of which are $2 \times 2 \times 0.105 \mathrm{~m}$. The mesh size is $2 \times 1.25 \times 1.75 \mathrm{~cm}$ (horizontal $\times$ vertical $\times$ thickness). The brick and the mortar behaviour are simulated using the advanced PRM concrete material model [6]. Material parameters like the maximum compressive strength, the maximum tensile strength, the fracture energy, etc. are then fitted in order to reduce as much as possible the gap between numerical and experimental results. In these simulations, the mortar strength is assumed to be two times less than the brick one. Fig. 1 compares experimental and numerical results of this case at three different instants.
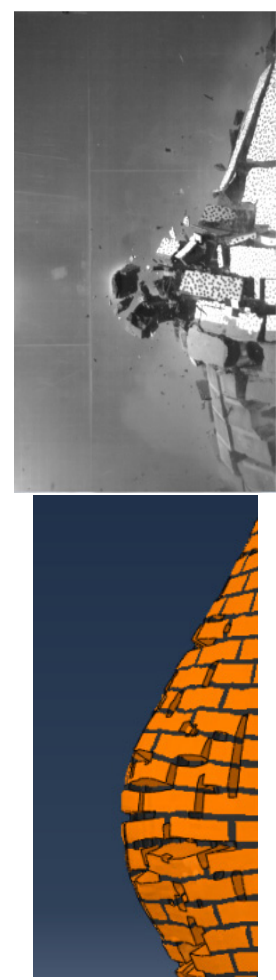

$25 \mathrm{~ms}$

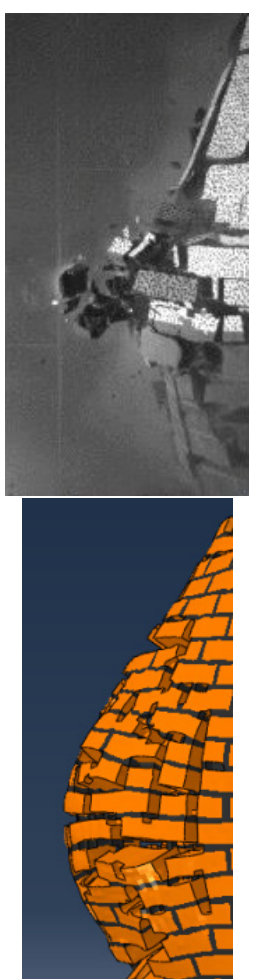

$30 \mathrm{~ms}$
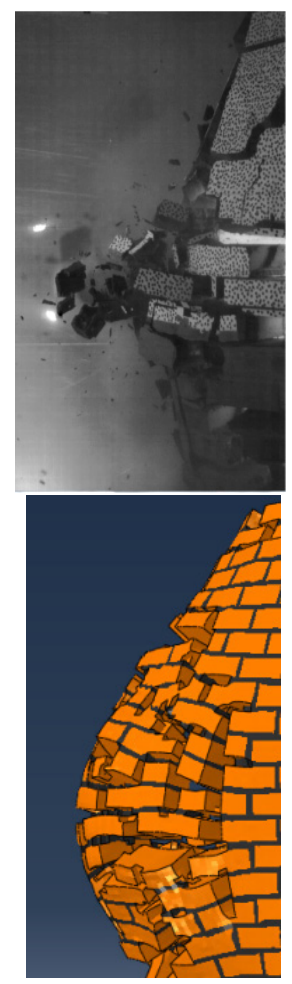

$35 \mathrm{~ms}$

Figure 1: Deflection of the back face of the wall. Comparison between experimental (upper part) and numerical (lower part), results obtained on a brick wall under blast effects. 
Using the fitted material parameters, FEM simulations are then carried out on brick walls loaded by a uniform and increasing pressure. A specific numerical procedure is used in order to prevent unstable structure response. Pressure loading is applied to the wall thanks to an incompressible fluid. A displacement is imposed on one side of the fluid while the other face exerts pressure on the wall in such a way that the wall is both subjected to uniform pressure and to controlled displacement. In this quasi-static numerical simulation, the applied pressure is plotted versus the wall deflection. Two quasi-static simulations are performed, one with a $0.105-\mathrm{m}$-thick thin wall which is the one used in the experiments and the other with a 0.210 -m-thick wall. The brick orientation is different for the thickest wall where the brick length corresponds to its thickness. In that case, the distance between two mortar joints is consequently lower in the horizontal direction. Fig. 2 shows the responses of both walls under the quasi-static loading. The curves on the lower part correspond to the evolution of the applied pressure versus the panel deflection, the red one accounts for the thicker wall and the blue one for the thinner. They are approximated by two linear and perfectly plastic curves (dashed curves) for which the maximum deflection has been chosen in order to conserve deformation energy, i.e. the areas under the dashed curves are equal to the areas under the solid ones.

These perfectly plastic curves are then used to identify the material parameters of eqns (1-3): the equivalent Young modulus $E$, the maximum strength $\sigma_{\max }$, the elastic deflection $x_{e}$ and the ductility $\delta$. In this particular case, a not obvious fact is that the material strength $\sigma_{\max }$ of the thin wall is by $29 \%$ higher than the one identified for the thick wall. This result can be interpreted considering the brick arrangement. As mentioned before, for the thick wall, vertical mortar joints are closer to each other and mortar has been assumed to be two times less resistant than brick. That is surely why the numerical material strength of the thick wall is comparatively weaker, a point that has not been experimentally checked yet.

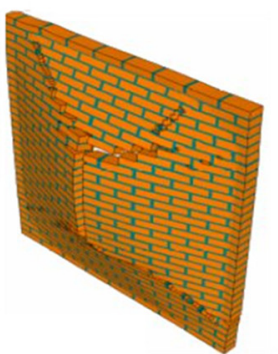

Thin wall $(0.105 \mathrm{~m})$

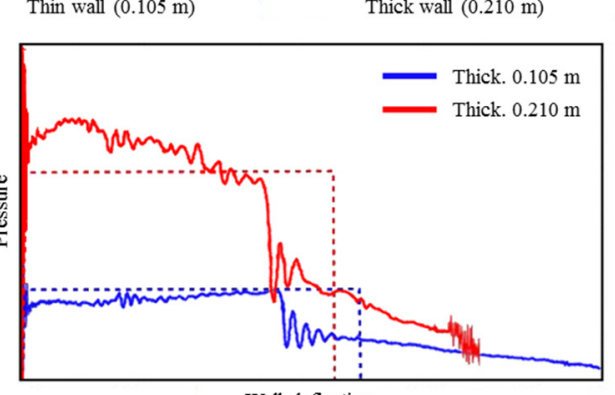

Wall deflection

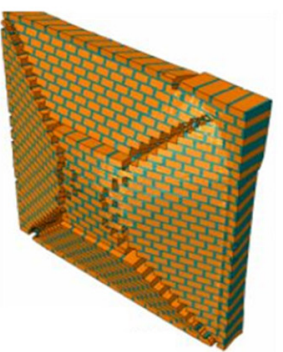

Thick wall $(0.210 \mathrm{~m})$

Figure 2: Numerical simulation of the response of plain brick walls under uniform pressure loading. Dashed curves account for elastic and perfectly plastic behaviour. 
One may regret the absence of additional experiments on identical brick walls but with different dimensions, border conditions and/or damage level to validate the parameters and the model. The significant cost of such a full experimental campaign explains this lacuna.

Finally, the identification procedure presented above can be applied for any other type of masonry walls such as stone, hollow brick, hollow concrete block, concrete, etc.

\subsection{Monte-Carlo method}

As indicated in the introduction, the result of a serious vulnerability study cannot be clear-cut because of the doubts about the problem characteristics. The Monte-Carlo method is a well-known means of propagating the input parameters' uncertainties through a model in order to quantify uncertainties in its results.

Let us consider on the one hand the $m$ parameters $\vartheta_{i}$ of the study including the model parameters, the distance between the explosion point and the masonry and, the hypothetical mass of the explosive charge. Consider on the other hand the material ductility $\delta$. Each of them is modelled as a random variable the form and characteristics of which are known or assumed (Normal, Uniform, etc.)

The Monte-Carlo method, illustrated in Table 1, consists in $n$ draws randomly exploring the hyperspace of the $m+1$ stochastic parameters (including $\delta$ ) and leading to $n$ computations each producing a value of $x_{\max } / x_{e}$. Statistics of the $n$ results can be analyzed regarding the statistics of $\delta$ as it will be exemplified in the next section.

It may be noted that $n$ must be large, typically between 1,000 and 1,000,000, in order to obtain statistically representative results. If computations are not fast enough to be performed in a reasonable time, their independence allows one to execute them simultaneously on multiple processing units.

Table 1: Scheme of a $n$-draws $m$-parameters Monte-Carlo experiment plan.

\begin{tabular}{|c|c|c|c|c|c|c|}
\hline \multirow{2}{*}{ Computation } & \multicolumn{5}{|c|}{ Parameters } & Result \\
\cline { 2 - 7 } & $\vartheta_{1}$ & $\vartheta_{2}$ & $\ldots$ & $\vartheta_{m}$ & $\delta$ & $x_{\max } / x_{e}$ \\
\hline$\# 1$ & & & $\ldots$ & & & \\
\hline$\# 2$ & & & $\ldots$ & & & \\
\hline$\ldots$ & $\ldots$ & $\ldots$ & $\ldots$ & $\ldots$ & $\ldots$ & $\ldots$ \\
\hline$\# n$ & & & $\ldots$ & & & \\
\hline
\end{tabular}

3 APPLICATION TO A MASONRY PANEL

\subsection{Study case}

As a first application, we consider a 3-m-height, 6-m-long and 0.22-m-thick plain-brick wall whose four borders are embedded. $\mathrm{An}_{2}-\mathrm{kg}$ exploding charge is located in front of the centre of the wall at a deterministic normal distance $d$ varying from 4 to $20 \mathrm{~m}$. The charge is supposed spherical.

All the parameters are stochastic and modelled as independent Normal (Gaussian) random laws: the three dimensions of the wall $L, l$ and $e$ and the failure stress $\sigma_{\max }$ have a standard deviation (StD) set to $10 \%$ of their mean value. In the case of $\rho$ and $E$ it is set to $5 \%$. Concerning the ductility $\delta, 10 \%$ has also been chosen. Histograms of the ten thousands draws, obtained with the Python package numpy.random, are displayed in Fig. 3. 


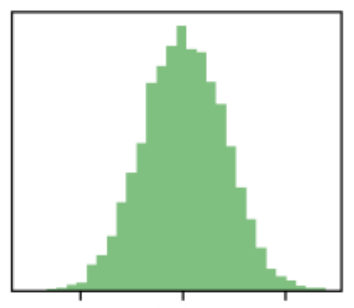

Failure stress $\sigma_{\max }(\mathrm{MPa})$

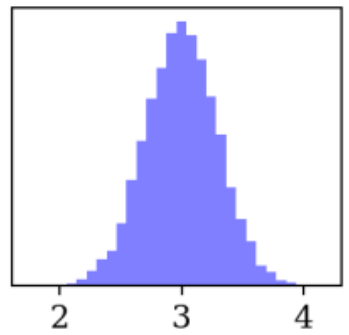

Height $l(\mathrm{~m})$

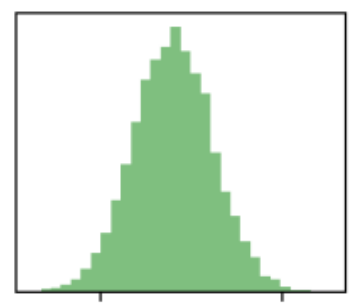

Density $\left(\mathrm{kg} / \mathrm{m}^{3}\right)$

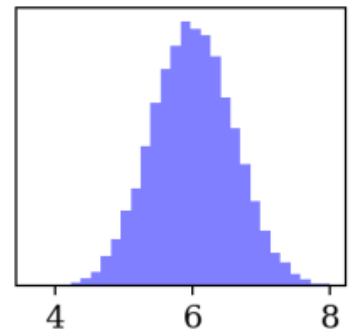

Width $L(\mathrm{~m})$

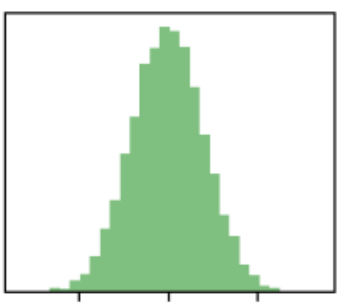

Young's modulus $E(\mathrm{GPa})$
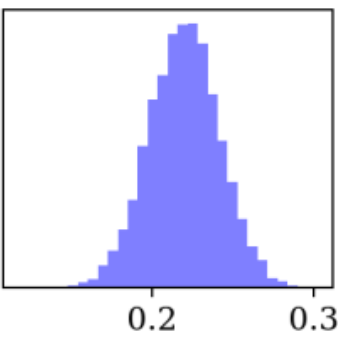

Thickness $e(\mathrm{~m})$

Figure 3: Normalized histograms of the six stochastic parameters of the model $(10,000$ draws). Values for material parameters (first line) are voluntarily not displayed.

\subsection{Results and analysis}

The 10,000-iterations Monte-Carlo simulations are performed for different charge - wall distances between 4 and 20 meters with a 0.5 -m-step. Histograms of $x_{\max } / x_{e}$ are converted into probability density functions (pdf) thanks to the Gaussian kernel density estimator from scipy.stats, using the Scott's bandwidth method [7]. These pdfs (grey) are compared to the ones of $\delta$ (red) as shown in Fig. 4 for selected distances. The probability of failure of the wall for any value $\Delta$ of $\delta$ (percentiles or maximum likelihood) is trivially obtained integrating the pdf of $x_{\max } / x_{e}$ where $\frac{x_{\max }}{x_{e}} \geq \Delta$. Titles of Fig. 4 give the most plausible probability of failure for each distance along with the confidence interval (CI) at 95\%. For example, at 12 meters, there is an excellent confidence $(95 \%)$, given the problem data, that the failure probability might be between $3 \%$ and $23 \%$.

As displayed in Fig. 5, those results can be summarized as a hysteresis diagram of failure probability $v s$ distance for the $95 \%$ confidence interval. For comparison, the binary result obtained with the deterministic method, for which each parameter takes its mean as unique value, is also shown (black line). Note that the width of the hysteresis is mainly governed by the material ductility standard deviation whereas its slope relies to the standard deviations of all parameters (this point will be established in the next section). Regarding building vulnerability against terrorist attacks, it may help to identify at which distance an explosive charge presents a significant risk, contributing to defining the extent of potential high-security areas. Nevertheless, in this particular case, the destiny of the wall is not guaranteed between 6 and $16 \mathrm{~m}$, a quite important range of uncertainty that could be reduced thanks to a better insight of the parameters. 

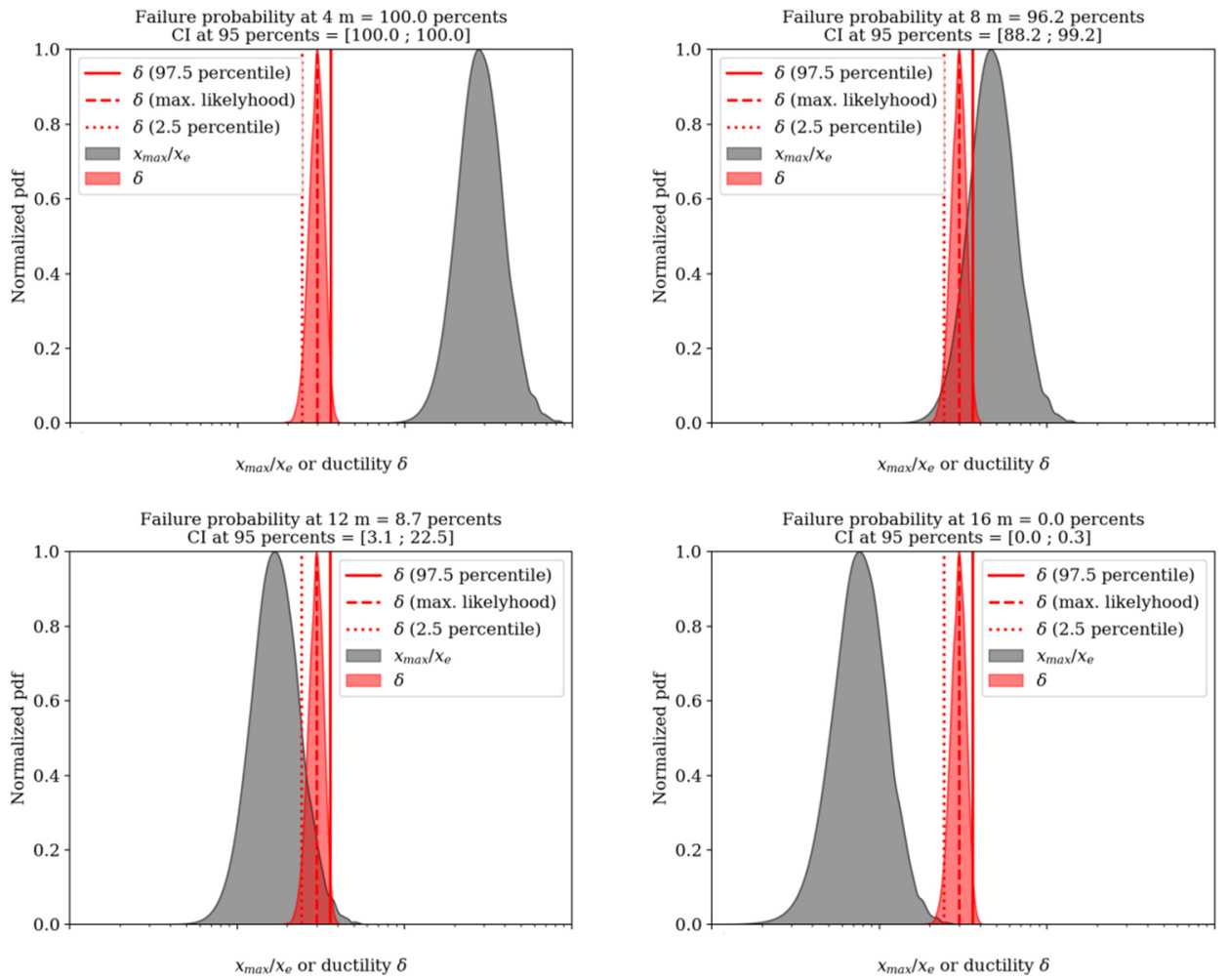

Figure 4: Monte-Carlo simulations for distances 4, 8, 12 and $18 \mathrm{~m}$. Grey area: pdf of $x_{\max } / x_{e}$. Red area: pdf of $\delta$ (values not displayed). Note the log-scale on x-axis.

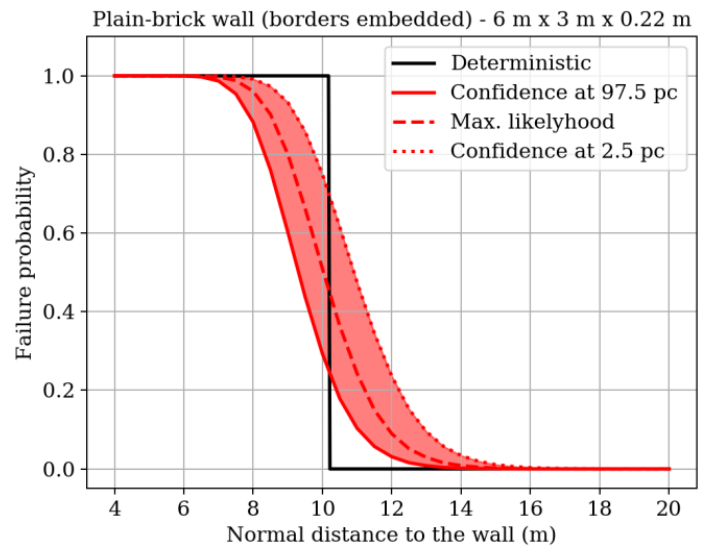

Figure 5: Summary of the Monte-Carlo simulations at distances between 4 and $20 \mathrm{~m}$. Confidence levels are given according to the failure point of view (pov) in opposition to the survival one. Red area denotes the failure probability within the $95 \%$ confidence interval. For comparison, the deterministic result is also displayed (black). 


\subsection{Sensitivity analysis}

Up to this section, lump uncertainties on geometrical and physical parameters have not been discussed even though they are directly responsible of the general trends of Fig. 5. And yet, it might be profitable to identify on which parameters efforts should be concentrated to significantly reduce uncertainties on wall destiny.

The sensitivity of the wall failure to the model parameters has been studied through Morris' sensitivity analyses [8] with the SALib package. Intervals of variation of the $\theta_{i}$ parameters were equal to their $95 \%$ confidence interval which are, since exclusively Normal laws have been used, $\left[\left(1-2 \tau_{i}\right) \dot{\theta}_{l} ;\left(1+2 \tau_{i}\right) \dot{\theta}_{l}\right]$ where $\dot{\theta}_{l}$ is their mean value and $\tau_{i}$ their standard deviation in percent. Given those intervals, Fig. 6 evidences at a distance of $10 \mathrm{~m}$, the dominant sensitivity of the wall thickness $e$ and the material strength and ductility $\sigma_{\max }$ and $\delta$. At other distances, results are qualitatively identical even though the respective sensitivities of the parameters are narrowed; a logical fact as $10 \mathrm{~m}$ is located at the heart of the hysteresis where results are the most indeterminate. It is noted that the sensitivity of parameters is only analysed on the binary outcome "failure" or "survival", meaning the effect of $\delta$ on the $95 \%$ CI of Fig. 5, i.e. the width of the hysteresis, is not considered.

Such results encourage enhancing the knowledge of the most sensitive parameters. As an illustration, Fig. 7 compares the original hysteresis of Fig. 5 to results produced after dividing by two the standard deviation $\tau_{i}$ of some parameters. As explained before, (b) and (d) demonstrate that $\delta$ strongly influences the width of the hysteresis. Besides, (c) demonstrates the effect of $e$ and $\sigma_{\max }$ on the slope but not on the width. Finally, a better insight into those three parameters led to a reduction from $(6 ; 16)$ to $(7.5 ; 13) \mathrm{m}$, the distance interval where the masonry panel destiny is not guaranteed. In the view of building protection, this is a key result for the definition of safety area. Obviously, it also could be valuable to reduce the uncertainties on other model parameters although they are less sensitive.

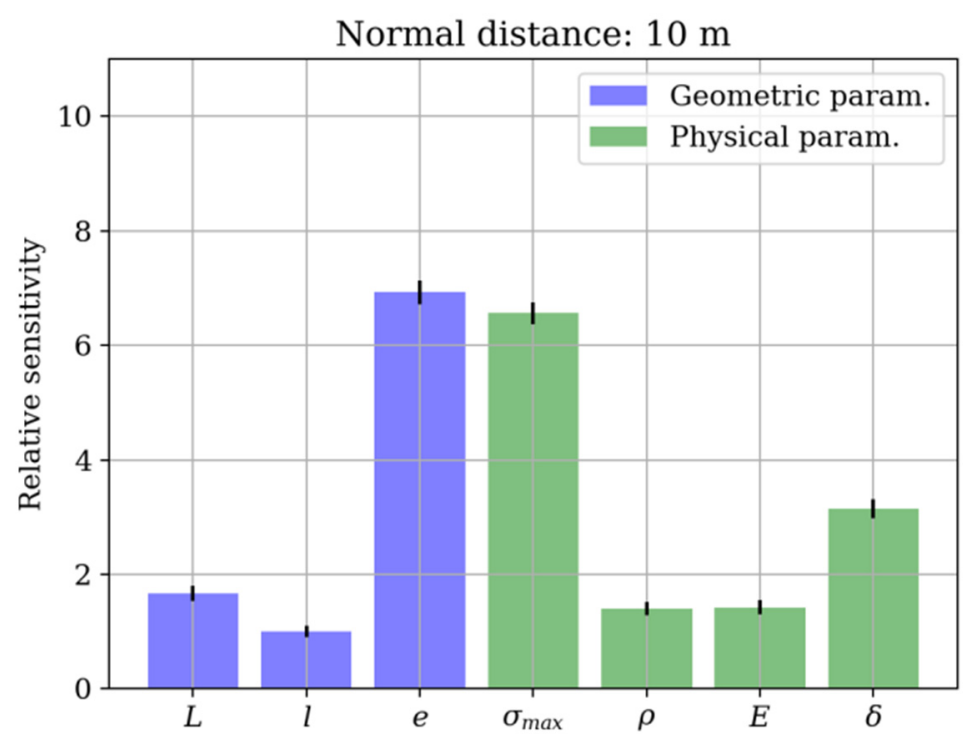

Figure 6: Morris' sensitivity analysis of model parameters on the wall failure/survival at $10 \mathrm{~m}$. Regarding their $95 \% \mathrm{CI}$, thickness $e$, strength $\sigma_{\max }$ and ductility $\delta$ reveal a high sensitivity. Scores of other parameters are lower and quite comparable. 


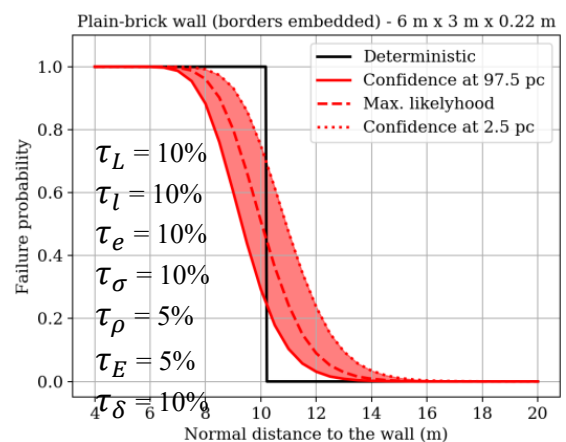

(a)

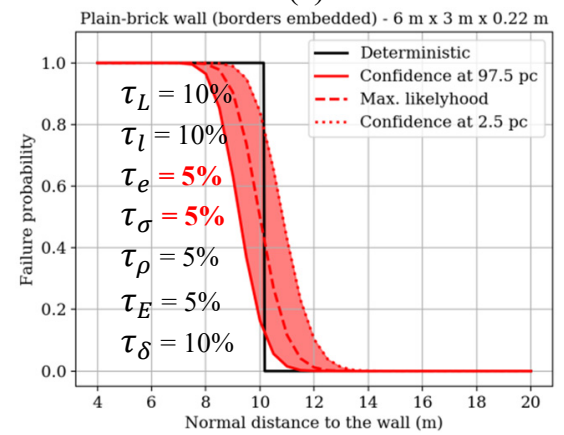

(c)

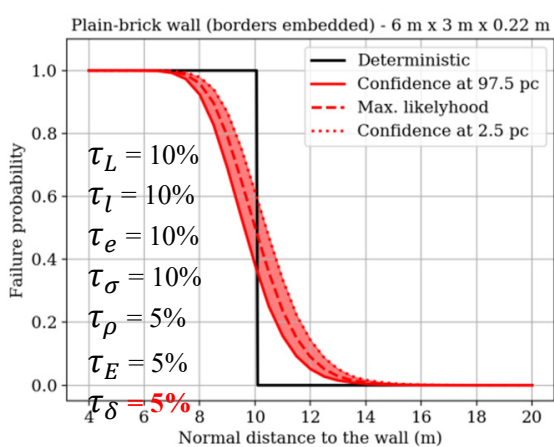

(b)

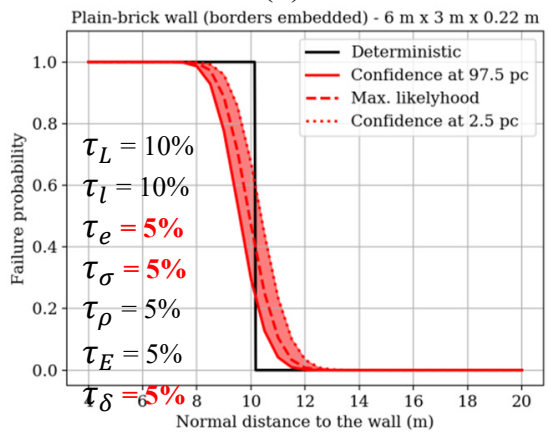

(d)

Figure 7: Summaries of the Monte-Carlo simulations at distances between 4 and 20 meters for different uncertainties on the model parameters. Red bold font: differences with reference. Confidence levels given according to the failure pov in opposition to the survival one. (a) Reference: standard deviations of section 3.2; (b) Effect of uncertainties on $\delta$; (c) Effect of uncertainties on $e$ and $\sigma_{\max }$; and (d) Effects of uncertainties on $e, \sigma_{\max }$ and $\delta$.

\section{APPLICATION TO BUILDINGS}

The generalization of our approach to buildings with multiple panels needed a better efficiency of the routines used previously. Thus, in the following, Python has been replaced by Java, which is a more highly performing language when numerous executions of the same routines are expected.

\subsection{Study case}

Consider a three-floor rectangular plain-brick building. Two frontages are composed of nine masonry panels and the two others are made of six. Each of them is in every respect the same as the one previously studied $(6 \times 3 \times 0.22 \mathrm{~m}$, see Section 3.1$)$. The roof is not modelled. Consider besides an explosive spherical charge of $\mathrm{M}_{2} \mathrm{~kg}$ located at an altitude of $1.5 \mathrm{~m}$ near the building. We are interested in two vulnerability analyses:

- The location of the charge is known and its capacity to damage the building has to be evaluated;

- A security perimeter against this threat has to be defined in order to avoid reasonable risk of damaging any part of the building. 


\subsection{Case of a known location}

Monte-Carlo simulation has been performed with 100,000 draws for each panel of the building, resulting in a total of 3,000,000 iterations. Partial results are shown in Fig. 8. The spherical charge appears in red and was located near a corner of the building at an altitude of $1.5 \mathrm{~m}$. A top view points out its exact location. Values and colours displayed on masonry panels of the $3 \mathrm{D}$ view correspond to the probability of failure $P_{f}$ for a confidence level of $97.5 \%$. It means there is only $2.5 \%$ of risk to underestimate the failure probability. It appears that mainly two walls are dangerously threatened $\left(P_{f} \sim 100 \%\right.$, purple), one seems to be moderately in peril $\left(P_{f} \sim 33 \%\right.$, green $)$ and all the others are almost out of danger $\left(P_{f}<5 \%\right.$, blue). Note that this result has been acquired within only about 35 seconds using one processing unit, a reasonable delay in an operational context.

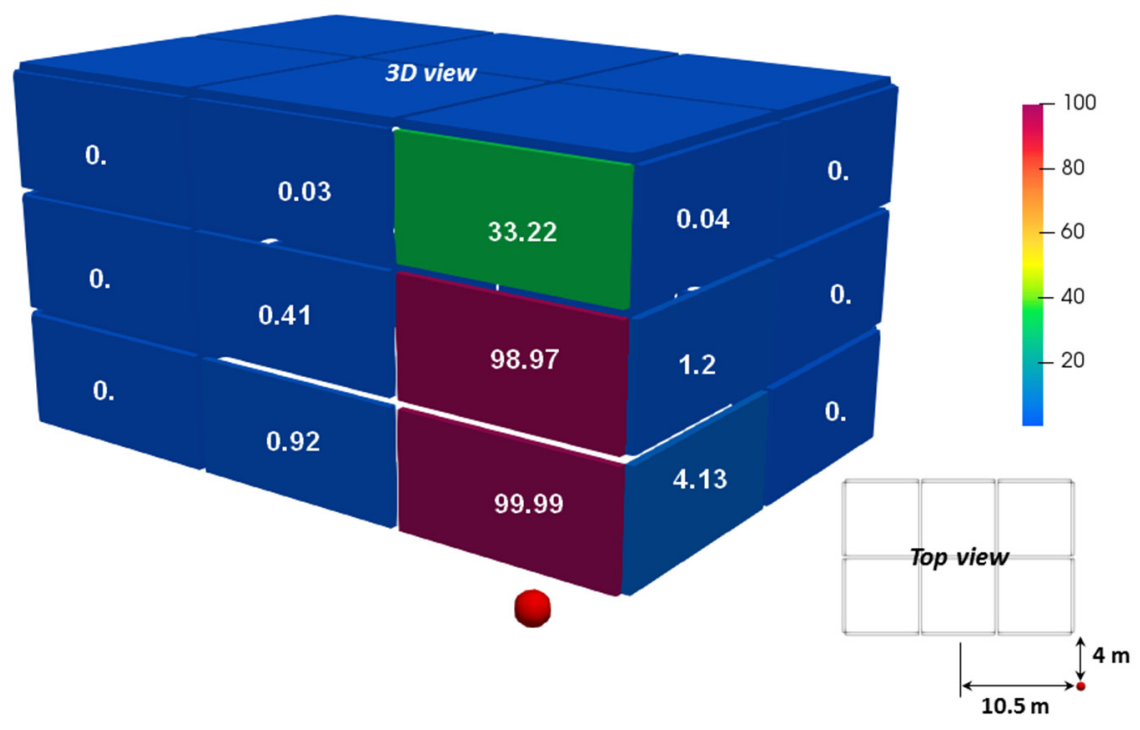

Figure 8: Failure probabilities (\%) of the masonry panels of a building submitted to the blast effect of a spherical $\mathrm{M}_{1}-\mathrm{kg}$ charge. Results given for a $97.5 \%$ confidence level meaning they have $2.5 \%$ of risk to be underestimated (survival pov).

\subsection{Case of the security perimeter}

Considering the same building, the same explosive charge and the same altitude of explosion, it might be convenient to define a security perimeter prior any real threat. Guided by the results of section 3.2, the geographic area to be considered around the building is between 4 and 20 meters from the frontages. This area is meshed in both directions every $0.5 \mathrm{~m}$ generating about 14,000 cells. For each cell, a Monte-Carlo simulation similar to the one of the previous section has been performed. Partial results are displayed in Fig. 9 as top-view colour maps where the six panels of the roof appear in grey. Colours account for the number of masonry panels of which $P_{f}$ is greater than a chosen value $P_{f}^{t h}$, according to the $97.5 \%$ confidence level (survival pov). Note that results are not exactly symmetrical because of the stochastic aspect of the Monte-Carlo method. 


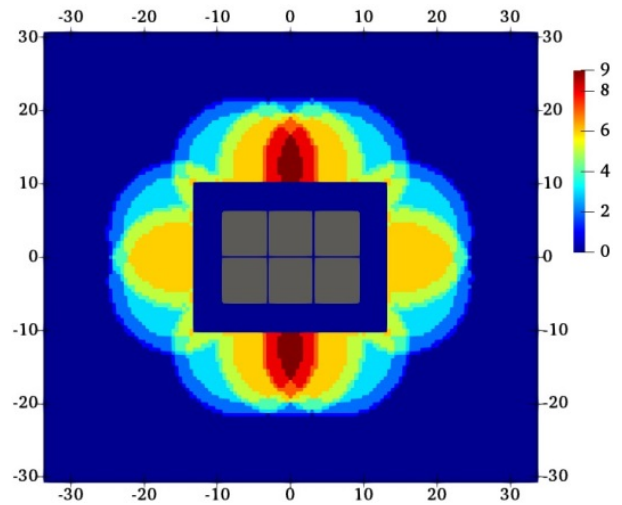

(a)

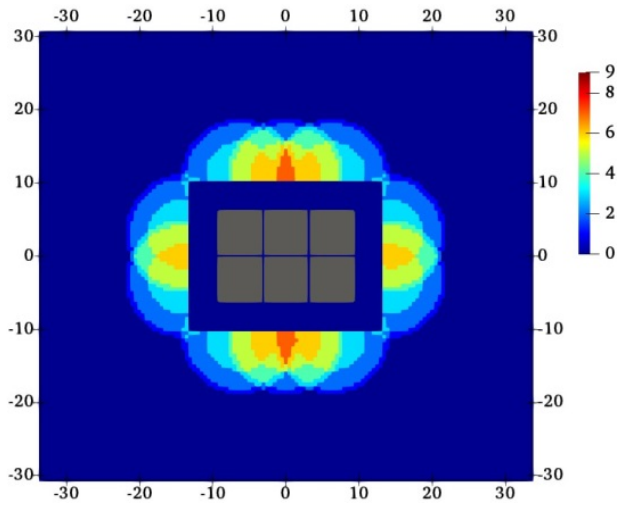

(b)

Figure 9: Number of panels for which $P_{f}>P_{f}^{\text {th }}$ according to the location of the explosion. (a) $P_{f}^{\text {th }}=1 \%$; (b) $P_{f}^{\text {th }}=20 \%$. Results given for a $97.5 \%$ confidence level according to the survival pov. Grey squares: roof panels.

Thanks to these colour maps, a security perimeter appears clearly depending on the accepted risk. For example, if no panel must be destroyed with a probability greater than $1 \%$, Fig. 9(a) indicates an exclusion area of around $45 \times 50 \mathrm{~m}$ while if this probability is set to $20 \%$, Fig. 9(b) specifies an area of $38 \times 45 \mathrm{~m}$. Obviously, a decrease of the confidence level would significantly decrease the area as well.

Computations leading to the results of Fig. 9, i.e. about 1.4 billion iterations, took about three and a half hours with 48 processing units running in parallel. It seems a negligible time as regards a context of training on securing strategic buildings.

\section{CONCLUSION}

Previous works led to modelling masonry panels as SDOF systems using analytical expressions. Such a fast-running engineering model (FREM) drastically decreased computation times of vulnerability studies compared to classical descriptive models. Experiments and FEM simulations helped to identify constitutive parameters of the FREM for various masonry materials.

This work aimed to use the uncertainties in material and geometric properties of masonry panels to get a better insight to the results of the model. The Monte-Carlo method has thus been used along with the Morris sensitivity analysis, both giving rich and complex information that has been clarified. Finally, a generalization of the approach to buildings with multiple panels has been conducted allowing advanced and time-efficient vulnerability studies either in emergency context or in case of safeguarding preparation.

Future work should consist in new experiments on brick walls as in Section 2.2 but with different dimensions, border conditions and/or damage level in order to validate the model and the parameters obtained in this study. Other masonry materials could also be investigated following our procedure. Additional work may concern the improvement of the SDOF model in order to take into account the presence of openings (windows, doors, etc.) on the masonry panel that, however, might not be a trivial study. Moreover, to consider other geometries than rectangular panels could be an interesting and useful work because of the irregular and particular shapes more and more used in modern western buildings. 


\section{REFERENCES}

[1] Timoshenko, S., Résistance des matériaux, première partie, théorie élémentaire et problèmes, Dunod: Paris, 1968.

[2] Timoshenko, S., Résistance des matériaux, deuxième partie, théorie développée et problèmes, Dunod: Paris, 1968.

[3] Benicourt, M., Charpente de navire, dynamique des structures. Analyse simplifiée, systèmes à un degré de liberté, Ecole nationale supérieure des techniques avancées, 1977.

[4] US Department of Defense, Structure to resist the effects of accidental explosions, UFC-3-340-02, 2008.

[5] Kinney, G.F. \& Graham, K.J., Explosive Shocks in Air, Springer-Verlag: Berlin, 1985.

[6] Pontiroli, C., Rouquand, A. \& Mazars, J., Predicting concrete behaviour from quasistatic loading to hypervelocity impact: an overview of the PRM model. European Journal of Environmental and Civil Engineering, 14(6), pp. 703-727, 2010.

[7] Scott, D.W., Multivariate Density Estimation: Theory, Practice, and Visualization, John Wiley \& Sons: New York, 1992.

[8] Morris, M., Factorial sampling plans for preliminary computational experiments. Technometrics, 33(2), pp. 161-174, 1991. 